\title{
Choline Phosphate Functionalized Cellulose Membrane Developed as Potential Hemostasis Dressing Based on a Unique Bioadhesion Mechanism
}

\author{
Xiaoqiang Yang ${ }^{1}, \mathrm{Na} \mathrm{Li}^{1}$, 2, Jayachandran N. Kizhakkedathu1, 3, Donald E. Brooks ${ }^{1,3^{*}}$ \\ ${ }^{1}$ Centre for Blood Research, 2350 Health Sciences Mall, University of British Columbia, Vancouver V6T 1Z3, \\ Canada. \\ ${ }^{2}$ School of Materials Science and Engineering, Zhengzhou University, Zhengzhou, China, 45000. \\ 3 Department of Pathology and Laboratory of Medicine, Room G227-2211 Westbrook Mall, University of \\ British Columbia, Vancouver V6T 2B5, Canada.
}

* Corresponding author. Tel.: +1-604-822-7085; email: don.brooks@ubc.ca

Manuscript submitted April 10, 2015; accepted June 25, 2015.

doi: 10.17706/ijbbb.2015.5.4.211-220

\begin{abstract}
A choline phosphate functionalized biocompatible cellulose membrane that can efficiently arrest human red blood cells was developed to have potential application in wound dressing. The bioadhesion is based on the unique multivalent electrostatic interaction between the head groups of phosphatidyl choline based lipids on the cell membrane and its inverse orientation but virtually identical structure, choline phosphate, coupled to the cellulose membrane. For functionalization, the cellulose membrane was decorated with the polymer brushes bearing multiple choline phosphate groups via surface-initiator atom transfer radical polymerization followed by the click chemistry. The molecular weight and the grafting density of polymer brushes grafted from the cellulose membrane surface were thoroughly evaluated by force-distance measurements using atomic force microscopy. The dependence on the number density of choline phosphate groups and the grafting density of human red blood cell binding to the cellulose membrane surface is reported.
\end{abstract}

Key words: Bioadhesion, cellulose membrane, choline phosphate, wound dressing.

\section{Introduction}

Traumatic hemorrhage is one of the most challenging problems for both military and civilian medicine despite early medical intervention and protective equipment. Uncontrolled hemorrhage remains the leading cause of mortality, accounting for $50 \%$ of all deaths on the battlefield [1] and $39 \%$ for civilian trauma deaths [2]. Generally, the traditional primary pressure-based methods for bleeding control of tourniquets, direct pressure, bandages, and clamping in most compressible cases, have not changed greatly in several centuries [3]. Hemorrhage dressing, as an alternative to bleeding control, which works by enhancing the clotting process has been developed and commonly utilized on the battlefield and clinical trials [4].

Absorptive wound dressing, one major category, is composed of multi-layer covers or single layer that provide either a semi-adherent quality or a non-adherent layer, combined with highly absorptive layers of fibers, such as cellulose, cotton or rayon. Generally, this type of dressing facilitates preparation of the wound bed with three types of action: capillary action, hydroconductive action and electrostatic action. Among 
these actions, electrostatic interaction is most commonly used as the action mechanism. For instance ChitoFlex ${ }^{\circledR}$ PRO hemostatic dressing [5], is fabricated from chitosan, a naturally occurring, bio-compatible polysaccharide which has a positive charge, it attracts red blood cells which have a negative charge. In the present work, a choline phosphate modified cellulose membrane was developed and can be potentially used as an absorptive wound dressing in the future. In our previous work [6], we described the discovery of a universal bioadhesion mechanism based on the interaction between the head group of phosphatidyl choline (PC)-lipids and the mirror image structure, choline 0-phosphate (CP). We found in fact that decorating a dendritic polymer, hyperbranched polygylcerol (HPG), with CP groups produces strong binding of the polymer construct to cell surfaces. Utilizing this concept, starting with a cellulose membrane (CM) we designed a grafting procedure based on growth of a polymer brush via atom transfer radical polymerization (ATRP)to which is coupled multiple CP groups using click chemistry, the goal being to use this material as substrate for a non-compressible hemorrhage dressing (Fig. 1) [7]. As hypothesized, the CP-bearing CM would have multivalent interactions with human blood, especially for the erythrocytes which have high concentrations of PC headgroup lipids in the cell membrane. We optimized our understanding of this system with compositional data and utilizing force-distance measurements by atomic force microscopy (AFM) to assess brush density determinants of binding and scanning electron microscopy (SEM) observation to measure cell binding. The goal is to use these constucts as potentially simple, but effective general tissue sealants in the future.

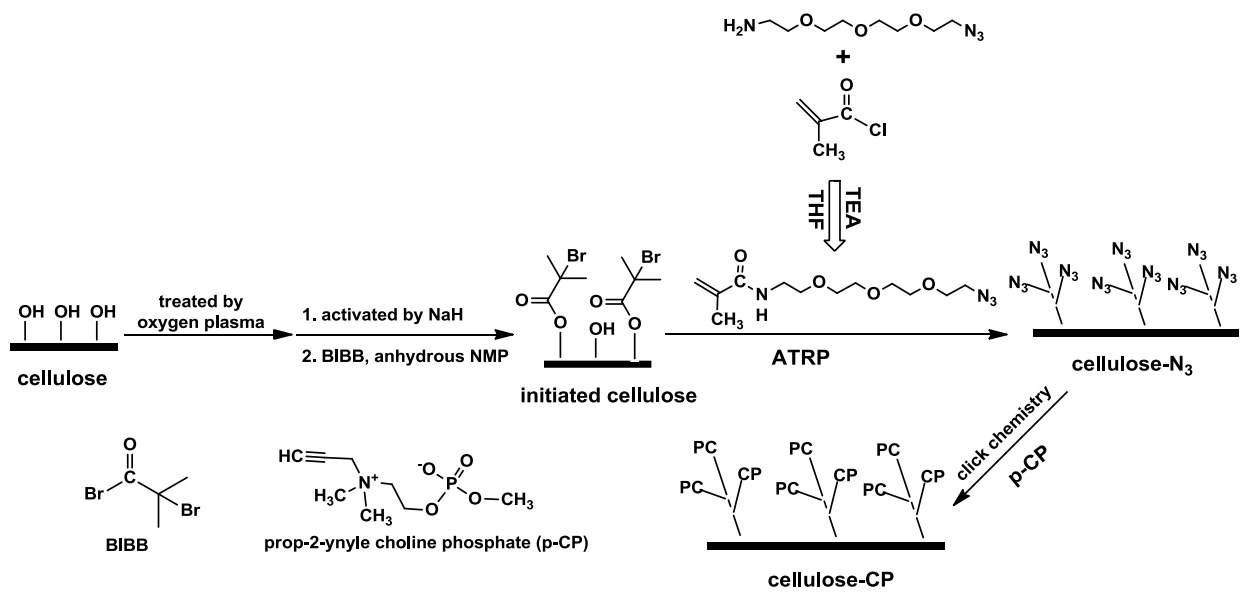

Fig. 1. Illustration of multiple CP-bearing CM via surface-initiated ATRP and click chemistry.

\section{Experimental Section}

\subsection{Materials}

Cellulose membrane (CM) (RC 55, RC 60, Whatman GmbH, Germany) was purchased from Fisher Scientific, CA; $\alpha$-Bromoisobutyryl bromide (BIBB, $98 \%$ ), anhydrous 1-methyl-2-pyrrolidinone (NMP, $99.5 \%)$, methacryloyl chloride (97 \%), copper (I) bromide (CuBr, $98.5 \%)$, 1,1,4,7,10,10-hexamethyltriethylenetetramine (HMTETA, $97 \%$ ), 3-dimethylamino-1-propyne (97\%), propargyl alcohol (99\%), 11-azido-3,6,9-trioxoundecan-1-amine (90\%) and trimethylamine (99\%) were purchased from Sigma-Aldrich (ON, CA). The 2-chloro-2-oxo-1, 3, 2-dioxaphopholate was purchased from Alfa Aesar. All the other chemicals were analytical reagents unless otherwise stated. Phosphate buffered saline (PBS, pH 7.4) and isotonic sodium chloride solution having ionic strength of $0.145 \mathrm{M}$ and $0.0145 \mathrm{M}$ were prepared in our lab. The whole blood was provided by the Centre for Blood Research from consenting volunteer donors. 


\subsection{Synthesis of ATRP Monomer N-(11-azido-3, 6, 9-trioxaundecanyl) Methacrylamide}

The building block, N-(11-azido-3, 6, 9-trioxaundecanyl) methacrylamide was obtained as a yellowy oil by the reaction of 11-azido-3, 6, 9-trioxoundecan-1-amine and methacryloyl chloride in the presence of triethylamine (TEA). Briefly, in a dried one-neck flask, $1.5 \mathrm{~g}(6.9 \mathrm{mmol})$ of 11-azido-3, 6, 9-trioxoundecan-1-amine was dissolved into the THF $(30 \mathrm{~mL})$, then $0.92 \mathrm{~g}(9.1 \mathrm{mmol})$ of TEA was added into the flask. Afterwards, the $0.8 \mathrm{~g}(7.6 \mathrm{mmol})$ of methacryloyl chloride was dropwise added to the mixture over $2 \mathrm{~h}$ under the ice-cooling condition, then the reaction left overnight at ambient temperature. The reaction mixture was filtered to remove the by-product followed by evaporation of the solvent. The obtained syrup was dissolved into dichloromethane (DCM) and washed with $0.1 \mathrm{M} \mathrm{HCl}$ solution and saturated $\mathrm{Na}_{2} \mathrm{CO}_{3}$ solution sequentially. The DCM was evaporated and a yellow oil was obtained as the crude product. The product was purified on a silica gel column using the mixture hexane and acyl acetate (15: 1) as the mobile phase.

\subsection{Synthesis of Prop-2-ynyle Choline Phosphate (p-CP) and Prop-2-ynyle Phosphatidyl Choline (p-PC)}

The synthesis of $\mathrm{p}$-CP and p-PC followed the recently reported method [6]. Briefly, for p-CP, double-distilled dimethylamino 2-propyne (25 g, $0.3 \mathrm{~mol})$, fresh anhydrous methanol ( $3.5 \mathrm{~g}, 0.11 \mathrm{~mol})$ and anhydrous acetonitrile $(60 \mathrm{~mL})$ were added by turning into a thoroughly flame dried Schlenk flask protected by Argon, and cooled to $-40{ }^{\circ} \mathrm{C}$ with dry ice/acetone solution. To the above mixture, double-distilled 2-chloro-2-oxo-1, 3, 2-dioxaphospholane (14.2 g, $0.1 \mathrm{~mol}$ ) was added dropwise, the reaction allowed to proceed for $2 \mathrm{~h}$ at $-40{ }^{\circ} \mathrm{C}$ and then gradually warmed to room temperature for another 4 $\mathrm{h}$. The precipitate was filtered off under argon atmosphere and the resulting mixture was stirred overnight at $65-70^{\circ} \mathrm{C}$. After reaction, the mixture was cooled down to RT and precipitated into THF. The precipitate was washed with THF until the filtrate solution was colorless. The solid was dried under vacuum and collected as a yellow waxy solid. As a control agent, the p-PC was synthesized following a similar procedure.

\subsection{Surface-Initiated ATRP from CM}

A two-step process of surface-initiated ATRP from CMs was performed (Fig. 1). In order to get the density of initiator on the surface of CM as high as possible, the CM substrates were firstly treated by oxygen plasma following a pre-optimized recipe to generate more hydroxyl groups on the surface to be reacted. Then the CMs were activated overnight by NaH solution in anhydrous N-methyl-2-pyrrolidone (NMP). After washing with NMP to remove the excess NaH under the protection of Argon, the activated CMs were esterified with BIBB ( $1 \mathrm{ml}$ for 3 pieces of CMs) in $20 \mathrm{~mL}$ of NMP solution at room temperature for 1 day. After reaction, the initiator functionalized CMs (CM-Br) were removed from the solution and thoroughly washed with methanol and water ultrasonically.

For the surface-initiated ATRP, three aliquots of CM-Br, $10 \mathrm{~mL}$ of methanol/water mixed solution $(1: 6 \mathrm{in}$ volume ratio) containing $20 \mathrm{mg}$ of HMTETA and a designed amount of monomer were introduced into a Schlenk flask followed by addition of $10 \mathrm{mg}$ of $\mathrm{CuBr}$ under Argon protection. The flask was then immersed in liquid nitrogen to freeze-thaw three times to degas and backfilled with Argon the last time of the cycle. The ATRP reaction proceeded at room temperature for predetermined time period, typically, 24 hour. After the reaction, the azide-functionalized $\mathrm{CMs}\left(\mathrm{CM}-\mathrm{N}_{3}\right)$ were removed from the solution, thoroughly washed with methanol and water ultrasonically, and dried under vacuum.

\subsection{Synthesis of CP/PC Functionalized CMs via Click Chemistry}

Typically, after introducing three pieces of $\mathrm{CM}-\mathrm{N}_{3}$ to a flask, $10 \mathrm{~mL}$ of mixed methanol/water $(4: 1 \mathrm{in}$ 
volume ratio) solution containing $10 \mathrm{mg}$ of $\mathrm{CuSO}_{4} \cdot 5 \mathrm{H}_{2} \mathrm{O}, 25 \mathrm{mg}$ of sodium ascorbate and $0.5 \mathrm{~g}$ of $\mathrm{p}$ - $\mathrm{CP}$ or $\mathrm{p}$-PC was added. The reaction was allowed to proceed at around $40^{\circ} \mathrm{C}$ for several hours. After reaction, the $\mathrm{CP} / \mathrm{PC}$ doped membrane (CM-CP or CM-PC) was washed with methanol and distilled water thoroughly under sonication, and dried at room temperature.

\subsection{Cell Binding Tests}

The CM substrates were placed in individual wells of 12 -well culture plates and first equilibrated with PBS for 10 mins, freshly prepared $5 \mathrm{ml}$ red blood cells (RBCs) were then added into each well and incubated at room temperature for $30 \mathrm{~min}$. After rinsing with PBS, the substrates were subjected to a series of graded alcohol-water solutions (70, 75, 80, 85, 90, 95 and $100 \%$ ) for 30 mins in each step, and dried at room temperature. Finally, the samples were examined under SEM; numerous locations were observed on each sample.

\subsection{Characterization Methods}

Nuclear magnetic resonance (NMR) was performed on a Bruker AV $400 \mathrm{MHz}$ NMR spectrometer at 300K. Surface functional characteristics were evaluated using attenuated total reflectance Fourier transform infrared (ATR-FTIR): the spectrum was collected on a Thermo-Nicolet Nexus FT-IR spectrometer with a MCT/A liquid nitrogen cooled detector, $\mathrm{KBr}$ beam splitter and MKII Golden Gate single refection attenuated total reflectance accessory. The Mass Spectrum was performed on a Waters ZQ equipped with ESCI ion source. Waters 2695 HPLC is used to deliver the samples. Atomic force microscopy (AFM) measurements were performed on a multimode, Nanoscope IIIa controller (Digital Instruments, Santa Barbara, CA) equipped with a fluid cell. Cantilevers were V-shaped silicon nitride with a tip radius of 5-40 nm and a spring constant of $0.06 \mathrm{~N} / \mathrm{m}$ as quoted by the manufacturer. The force measurements were performed in force mode and in a $10 \mathrm{mM} \mathrm{NaCl}$ solution; the samples were allowed to equilibrate for 15 mins before performing measurements. The onset of the region of constant compliance was used to determine the zero distance, and the region in which force was unchanged was used to determine the zero force. All measurements were taken at an approach rate of $1.0 \mathrm{~Hz}$. Each sample was measured in three different spots. The raw AFM force data were converted into the reduced force vs separation following the principle of Ducker et al. [8].

\section{Results and Discussions}

\subsection{Steps of Modification of Cellulose Surface}

In order to obtain more density of higher initiator surface density on the CMs they were first treated with oxygen plasma which is an efficient way to achieve more reactive hydroxyl groups [9]. The initiator of ATRP was immobilized on the sodium hydride activated cellulose membrane surface through esterification of hydroxyl groups with bromoisobutyryl (BIBB) in the anhydrous solvent of N-methyl-2-pyrrolidone (NMP), as shown in Fig. 1. The hydroxyl groups on the CMs were converted into cleavable tertiary bromoester groups, which are efficient activators for acrylate [10]. The grafting was accomplished by ATRP via a wet chemical method, e.g. immersing the initiator-modified CMs (hereafter refer to $\mathrm{CM}-\mathrm{Br}$ ) into the reaction mixture of methanol and water containing the monomer (N-(11-azido-3, 6, 9-trioxaundecanyl) methacrylamide), $\mathrm{Cu}(1) \mathrm{Br}$ and HMTETA; a sacrificial initiator, methyl $\alpha$-bromoisobutyrate, was also introduced to control the length of the grafting polymer [11]. The ATRP monomer, N-(11-azido-3, 6, 9-trioxaundecanyl) methacrylamide (ATMA), which has a non-cleavable amide bond was synthesized by the acrylation of 11-azido-3, 6, 9-trioxoundecan-1-amine with methacryloyl chloride, and was characterized and the structure confirmed by ${ }^{1} \mathrm{H}$ NMR and mass spectrometry $(\mathrm{m} / \mathrm{z} 309.16(\mathrm{M}+\mathrm{Na})$ (data not shown), the theoretical molecular weight is 286.33). The polymerization was carried out at room temperature for 
over $24 \mathrm{~h}$. After washing thoroughly under sonication, the samples were first characterized by ATR-FTIR and contact angle measurement. The pristine $\mathrm{CM}$ is quite hydrophilic while the initiator is hydrophobic. As the result, the contact angle of $\mathrm{CM}-\mathrm{Br}$ sharply increased to $118^{\circ} \pm 6$. However, the contact angle of $\mathrm{CM}-\mathrm{N}_{3}$ decreased again to $0^{\circ}$ after grafting the polymer on the surface via ATRP, showing a transformation from hydrophilic to hydrophobic, then back again to hydrophilic. The ATR-FTIR spectra shows the characteristic strong amide I absorption band and the stretching vibration of $\mathrm{N}_{3}$ observed at c.a $1640 \mathrm{~cm}^{-1}$ and $2100 \mathrm{~cm}^{-1}$ respectively after ATRP polymerization (Fig. 2(A)), confirming successful growth of polymer brushes on the surface. Moreover, the intensity of these bonds shows monomer concentration dependence, indicating the thickness/molecular weight increased with the monomer concentration (Fig. 2(B)).

The click reaction was used to couple the p-CP monomer to $\mathrm{CM}-\mathrm{N}_{3}$ to achieve multivalency for cell binding. The reaction was carried out at $\sim 40^{\circ} \mathrm{C}$ overnight. As shown in Fig. 2(C), the ATR-FTIR signal of the azido group disappeared while the new signal at $1207 \mathrm{~cm}^{-1}$ appeared which corresponds to phosphonate, indicating the complete click reaction and conversion from azido groups to CP groups. Meanwhile, the PC functionalized cellulose membrane (CM-PC) for control was prepared following the same method, and the structure was confirmed by the ATR-FITR, ${ }^{1} \mathrm{H}$ NMR and ${ }^{31} \mathrm{P}$ NMR as well (data not shown).
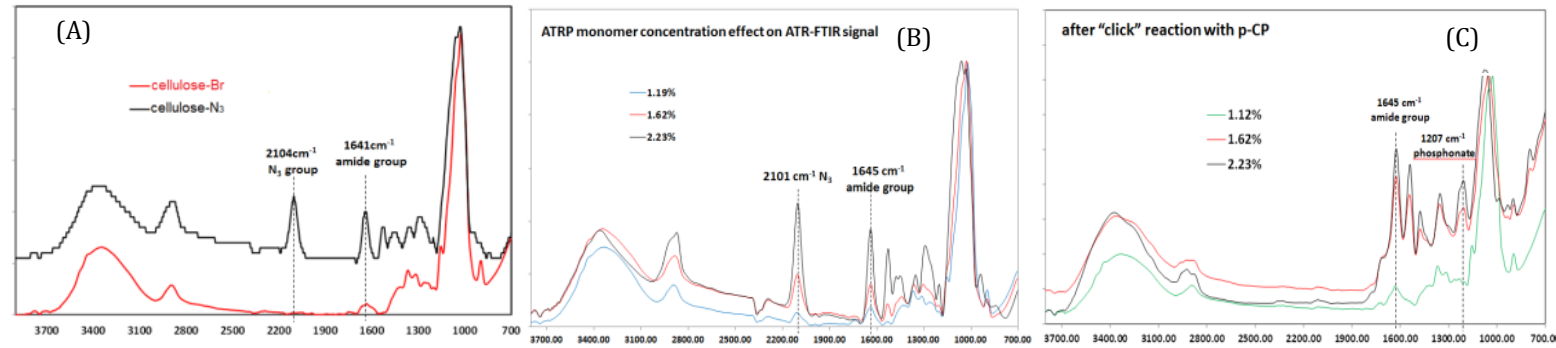

Fig. 2. ATR-FTIR spectra of comparison between cellulose-Br and cellulose-N3 (A), cellulose-N3 with different polymerized monomer concentration (B) and their corresponding cellulose-CP after "click" reaction $(\mathrm{C})$.

\subsection{Calculate the " $k$ " Value Using Silicon Wafer Model}

The graft density, one of the key characteristics of polymer brushes, is determined by the distance $(D)$ between grafting points, and can be calculated based on the dry thickness $(h)$ and molecular weight $\left(M_{n}\right)$ of grafted polymers. However, it is difficult to measure the thickness of a polymer brush on a rough cellulose membrane by ellipsometry and calculate the graft density. According to the Alexander Model for the theoretic description of polymer brushes, the wet brush height scales linearly with the degree of polymerization/molecular weight of the polymer molecules when the grafting density is known. Hence, we adopt the equation obtained for brushes in a good solvent to calculate the graft density indirectly. That is:

$$
h=k N \times \sigma^{1 / 3}
$$

where $h$ is the brush thickness in solvent, $k$ is the length of methacrylamide monomer, $N$ is the degree of the polymerization, and $\sigma$ is the grafting density. If we know the " $k$ " value for this particular polymer molecule, and measure the wet thickness $(h)$ and molecular weight through AFM, then we can calculate the graft density by the equation (1). To obtain the " $k$ " value, a series of polymers grafted from silicon wafers were prepared via SI-ATRP using the same monomer under similar conditions as for he cellulose membrane. The graft density $(\sigma)$ of each sample was calculated using the equation: 


$$
\sigma=\frac{h \rho N_{\mathrm{A}}}{M_{n}}\left(\rho=1.05 \mathrm{~g} / \mathrm{cm}^{3}\right)
$$

where $h$ is the dry thickness measured by ellipsometry, $\rho$ is the density of polymer, $N_{\mathrm{A}}$ is the Avogadro constant. $M_{n}$ is measured by GPC from cleaved polymer on the surface. As a result, the " $k$ " value of this monomer was linearly fitted as the slope of equation (1) giving $k=0.51 \mathrm{~nm}$, which was similar to the reported average length of an amino acid, $0.4 \mathrm{~nm}$ [12]. The corresponding characteristic properties of polymer brushes on the surface of CMs are listed in Table 1. It is seen from the table that all the grafted polymers (S1-S4) on the CMs form polymer brushes not mushroom shaped, which was evidenced by the $D / R_{\mathrm{g}}<2$.

Table 1. Characteristic Properties of Polymer Brushes on the Cellulose Membrane

\begin{tabular}{ccccc}
\hline \hline samples & $h_{\text {wet }^{\mathrm{a}}}$ & $M_{n, \mathrm{AFM}^{\mathrm{b}}}$ & $\begin{array}{c}\text { graft density }(\sigma)^{\mathrm{c}} \\
\left.\text { (chains } / \mathrm{nm}^{2}\right)\end{array}$ & $\mathrm{D} / R_{\mathrm{g}} \mathrm{d}^{\mathrm{c}}$ \\
\hline S1 & 36 & 30,900 & 0.28 & 0.63 \\
S2 & 15 & 12,500 & 0.31 & 0.59 \\
S3 & 27 & 21,700 & 0.35 & 0.56 \\
S4 & 37 & 27,400 & 0.44 & 0.50 \\
S5 & 37 & 47,500 & 0.08 & 1.18 \\
S6 & 31 & 45,000 & 0.06 & 1.36 \\
S7 & 21 & 48,000 & 0.02 & 2.35 \\
\hline \hline
\end{tabular}

a $h_{\text {wet: }}$ equilibrium thickness of polymer brush measured in hydrated state by AFM.

b $M_{n}$, AFM: molecular weight was calculated by the equation $M_{i} / M_{\text {mon }}=L_{c, i} / I(3)$ by AFM.

${ }^{c}$ grafting density $(\sigma)$ was calculated by the equation (1).

${ }^{\mathrm{d}} R_{\mathrm{g}}$ denotes radius of gyration of polymer, measured by GPC.

\subsection{Surface Analysis by AFM}

The AFM is best known for its high-resolution imaging capabilities [13]-[14], but it is also a powerful tool for sensitive force-distance measurements [15]-[16]. Fig. 3 shows the representative force curves (both approach and retraction) between the tip and different CM surfaces in ionic aqueous medium. Because of its hydrophilic pristine surface, there was no hysteresis for CM between the approach and withdrawal curves for the bare material (Fig. 3(A)). However, when the hydrophobic ATRP initiator was anchored onto the CM surface, much more interaction was observed. As shown in Fig. 3(B), in the approach curve, the jump-to-contact (JTC) of the cantilever, an effect referred to as "snap-in" was observed when the tip came close to the surface. When pulling off, a much stronger adhesive force was observed $\left(F_{\text {adhesive }}=1.1 \pm 0.2 \mathrm{nN}\right)$. After grafting polymer, the approach curve of $\mathrm{CM}-\mathrm{N}_{3}$ exhibits a repulsive force instead of a JTC, to confirm the polymer brushes were successfully grafted on the surface.
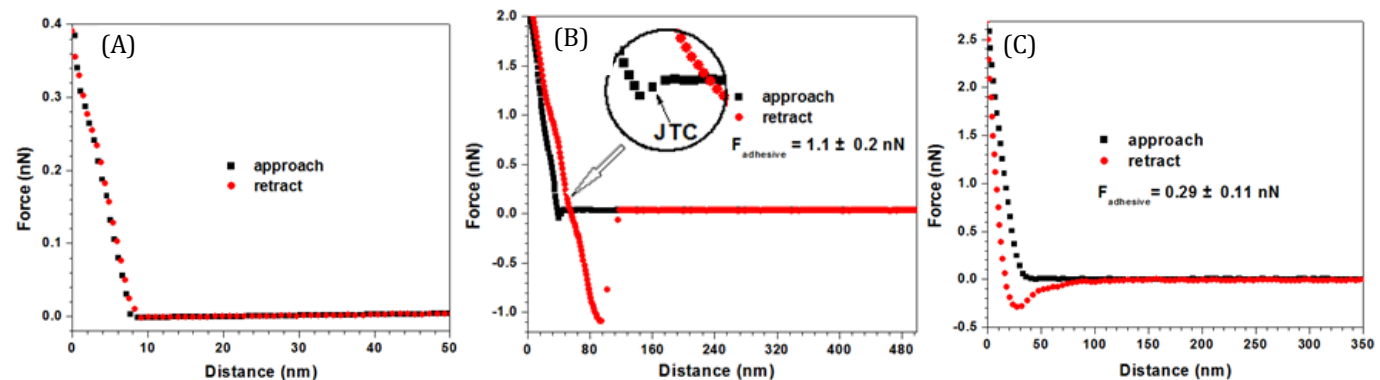

Fig. 3. Representative force curves of cellulose control (A), cellulose- $\mathrm{Br}(\mathrm{B})$ and cellulose- $\mathrm{N}_{3}(\mathrm{C})$. 


\subsection{Effect of the Polymer Weight of Polymer Brush on Cell Binding}

\subsubsection{Molecular weight of polymer brush evaluation by AFM}

It is reported that the higher the ATRP monomer concentration, the higher the molecular weight of grafted polymer for surface-initiated ATRP. In order to investigate the molecular weight of grafted polymer's (e.g. the number of $\mathrm{CP}$ groups) effect on cell binding, we prepared different $\mathrm{CM}-\mathrm{N}_{3}$ samples with varied molecular weight of polymer brushes while keeping the grafting density consistent via the ATRP reaction. To do this, ATRP was carried out with 1.19, 1.62, and 2.23 wt \% (refer to S2, S3 and S4, respectively in Table 1) monomer concentration respectively at room temperature for $24 \mathrm{~h}$ for a given copper catalyst concentration. The molecular weight $\left(M_{n, \text { AFM }}\right)$ of each sample was calculated based on our previously established method using the following equation:

$$
M_{i} / M_{\mathrm{mon}}=L_{\mathrm{c}, i} / I
$$

where $M_{\text {mon }}$ is the molar mass of the monomer, $I$ is the size of the monomer backbone, $0.25 \mathrm{~nm}$ and $L_{c, i}$ is the chain contour length of the portion of the chain being extended. However, we calculated the $M_{n \text {, AFM }}$ according to the above equation by taking the separation $L_{\mathrm{m}}$ as an estimate for the average contour length. $L_{\mathrm{m}}$ is the distance over which the attractive force increases with extension and reaches a maximum value. The equilibrium brush height of each sample was measured from the approach curve and obtained as an average of 10 force profiles. As a result, the $M_{n, \text { AFM }}$ of the grafted polymer chains for the S2, S3 and S4 was $1.25 \times 10^{4}, 2.17 \times 10^{4}$, and $2.74 \times 10^{4} \mathrm{Da}$, respectively. Moreover, the maximum attractive force $F_{\mathrm{m}}$ measured by AFM was $0.24 \pm 0.08 n \mathrm{~N}$ for the sample with $M_{n \text {, AFM }}$ of $12.5 \mathrm{KDa}, 0.29 \pm 0.09 n \mathrm{~N}$ for $M_{n, \mathrm{AFM}}$ of $21.7 \mathrm{KDak}$, and $0.25 \pm 0.07 \mathrm{nN}$ for $M_{n, \mathrm{AFM}}$ of $27.4 \mathrm{KDa}$. The slight difference of maximum attractive force shows that the estimated molecular weight of polymer brush is independent of the force when the polymer brushes are dense.

\subsubsection{RBC binding dependence on the polymer brush molecular weight}

Click chemistry after controlled ATRP was carried out to convert the azido group on the CM surface to the CP group. We assume the conversion was $100 \%$ which was confirmed by the aforementioned ATR-FTIR characterization. The individual CM-CP and the control samples (blank and CM-PC) were incubated with freshly prepared RBCs (incubated in PBS $15 \mathrm{~min}$ before exposure to CMs) for $30 \mathrm{~min}$ at room temperature. Fig. 4 shows the SEM images of CM-CP with varied molecular weight after contact with RBCs. The CM-CP which has the $M_{n, \text { AFM }}$ of $12.5 \mathrm{KDa}$, with an estimated $44 \mathrm{CP}$ groups $\left(M_{n, \mathrm{AFM}} / M_{\text {repeat unit }}\right)$ decorated on the surface, shows little RBC binding effect (Fig. 4(A)). However, when the CP groups are increased to 76 and further to 96, the CM-CP shows much stronger binding of RBCs. As shown in Fig. 4(B) and Fig. 4(C), the RBCs almost fully covered the surface and were dispersed uniformly, almost forming a complete layer. The $\mathrm{RBC}$ shape distortion occurs due to the adhesive forces overcoming the inherent elasticity of the composite cell membrane structure. This observation is consistent with our previous finding on the effect of changing the CP content of HPG-CP on binding to RBCs [6]. When the RBCs came in contact with the original cellulose membrane and PC-decorated membrane, negligible cell binding was observed, clearly indicating the specificity of the CP-based reaction with the cell surface (data not shown).

\subsection{Effect of the Grafting Density of Polymer Brush on the Cell Binding}

CM-N $\mathrm{N}_{3}$ surfaces having similar $M_{n, \text { AFM }}$ but with different grafting density $\sigma$ were obtained by hydrolyzing a polymer brush with high $\sigma$ for an extended period in $1 \mathrm{M} \mathrm{NaOH}$ solution providing different $\sigma$ values at different hydrolysis times. The molecular weight of each sample was confirmed as roughly identical which 
was around $45 \mathrm{kDa}$ measured by AFM. As shown in Fig. 5(A), a strong and tightly sealed layer formed on the surface with high brush grafting density of polymer brush decorated with CP groups. Using equation (1) for $\sigma$ and assuming $\mathrm{CP} /$ polymer we estimate there were $168 \mathrm{CP}$ groups per mol anchored on the surface providing multivalent interaction sites and binding energy for the RBCs. The RBC shape distortion shows evidence of strong electrostatic interactions as well. Comparing the cell binding effect of CM-CP with the lower molecular weight but higher grafting density (for instance, the sample with $\sigma$ of 0.44 and $M_{n \text {, AFM }}$ of 27.4 kDa for Fig. 4(C), S4 in Table 1), it seems the molecular weight of the polymer brushes, i.e. the CP density, has more effect on the cell binding even if the grafting density is relatively low. When the grafting density was reduced to 0.08 after $17 \mathrm{~h}$ hydrolysis (S6 in Table 1), there were still crowded RBCs fully covering the surface although the shape recovered and became pristine (Fig. 5(B)). With the hydrolysis process proceeding, the grafting density was lower to 0.02 (S7 in Table 1), at this point, the grafted polymer belongs to the mushroom regime due to the $D / R_{\mathrm{g}}>2$, fewer RBCs were binding to the surface and more cellulose substrate was exposed (Fig. 5(C)). Finally, the polymer brush was cleaved completely from the surface under the hydrolysis condition, and occasional single RBCs adhered to the surface, indistinguishable from the cellulose control sample (Fig. 5(D)).

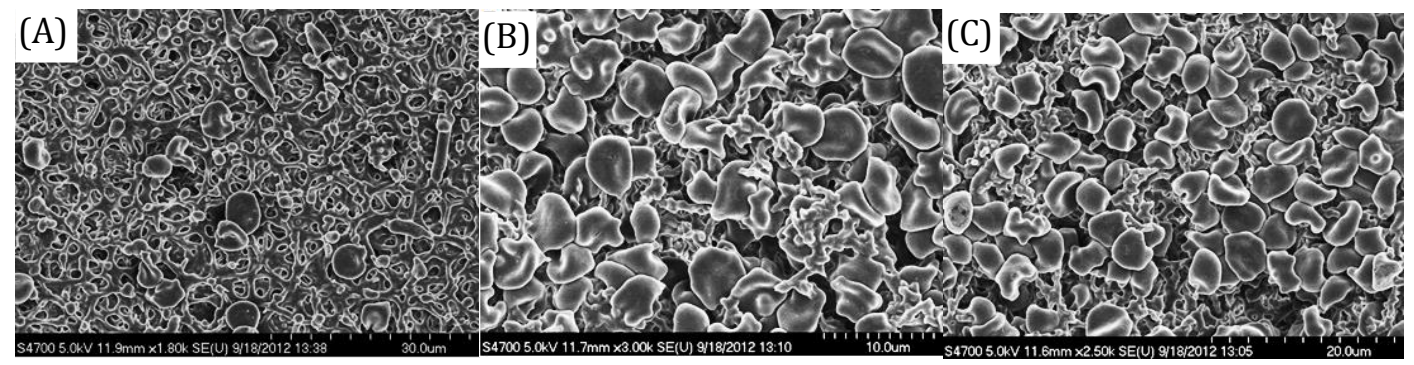

Fig. 4. SEM observed RBC incubated with (A) cellulose-CP with $M_{n, \text { AFM }}$ of $12.5 \mathrm{KDa}$; (B) cellulose-CP with $M_{n, \mathrm{AFM}}$ of $21.7 \mathrm{KDa}$; (C) cellulose-CP with $M_{n, \mathrm{AFM}}$ of 27.4 KDa.
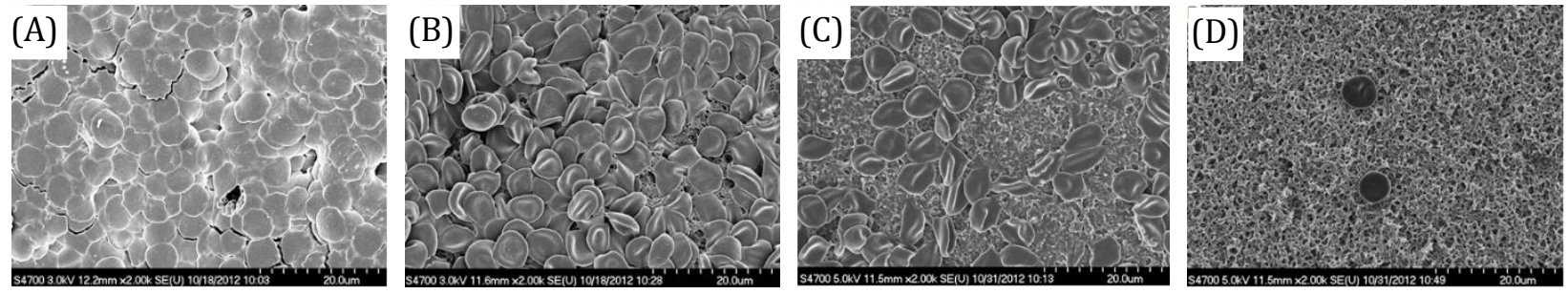

Fig. 5. SEM micrographs of RBC incubated with cellulose-CP with same $M_{n \text {, AFM }}$ at variable hydrolysis time ((A) before hydrolysis; (B) after $17 \mathrm{~h}$ hydrolysis; (C) after 9 days hydrolysis; (D) complete hydrolysis).

\section{Conclusion}

In summary, we have presented a facile method for the preparation of choline phosphate-decorated cellulose membranes that in principle can be used as an effective hemostasis dressing. The CM-CP was synthesized via multi-step modifications including surface-initiated ATRP and click chemistry. The modified CMs were characterized by ATR-FTIR and especially by the force measurements with AFM. The short-range interactions between the grafted polymer brush and AFM tip were evaluated appropriately to provide values for the molecular weight and grafting density of the polymer brushes. We showed that multiple valence electrostatic interactions were responsible for binding the RBCs which presumably have PC groups adhering onto the CP-decorated cellulose membrane. Comparing the bioadhesion's dependence on the CP density (e.g. molecular weight) and the grafting density of polymer brush on the surface, the binding effect 
relies less on the grafting density than on the molecular weight, the latter reflecting the higher $\mathrm{CP}$ surface density. In the future, we will further investigate other determining factors and develop a working membrane/pad for wound dressing.

\section{References}

[1] Bellamy, R. F. (1984). The causes of death in conventional land warfare: Implications for combat casualty care research. Military Medicine, 149(2), 55-62.

[2] Acosta, J. A., Yang, J. C., Winchell, R. J., Simons, R. K., Fortlage, D. A., Hollingsworth-Fridlund, P., et al. (1998). Lethal injuries and time to death in a level I trauma center. Journal of American College of Surgeons, 186(5), 528-533.

[3] Alam, H. B., Burris, D., DaCorta, J. A., \& Rhee, P. (2005). Hemorrhage control in the battlefield: Role of new hemostatic agents. Military Medicine, 170(1), 63-69.

[4] King, K., Neuffer, M. C., McDivitt, J., Rose, D., Cloonan, C. C., \& Vayer, J. S. (2004). Hemostatic dressings for the first responder: A review. Military Medicine, Academic Emergency Medicine, 169(9), 716-720.

[5] Littlejohn, L. F., Devlin, J. J., Kircher, S. S., Lueken, R., Melia, M. R. A., \& Johnson, S. (2011). Comparison of celox-A, chitoflex, woundstat, and combat gauze hemostatic agents versus standard gauze dressing in control of hemorrhage in a swine model of penetrating trauma. Academic Emergency Medicine, 18(4), 340-350.

[6] Yu, X. F., Liu, Z. H., Janzen, J., Chafeeva, I., Horte, S., Chen, W., et al. (2012). Polyvalent choline phosphate as a universal biomembrane adhesive. Nature Materials, 11, 468-476.

[7] Arnaud, F., Teranishi, K., Tomori, T., Carr, W., \& McCarron, R. (2009). Comparison of 10 hemostatic dressings in a groin puncture model in swine. Journal of Vascular Surgery, 50(3), 632-6399.

[8] Ducker, W. A., Sendan, T. J., \& Ashley, R. M. P. (1992). Measurement of forces in liquids using a force microscope. Langmuir, 8, 1831-1836.

[9] Bhattacharya, S., Datta, A., Berg, J. M., \& Gangopadhyay, S. (2005). Studies on surface wettability of poly (dimethyl) siloxane and glass under oxygen-plasma treatment and correlation with bond strength. Journal of Microelectromechanical Systems, 14(3), 590-597.

[10] Matyjaszewski, K., Gaynor, S. G., Kulfan, A., \& Podwika, M. (1997). Preparation of hyperbranched polyacrylates by atom transfer radical polymerization. 1 . Acrylic $\mathrm{AB}^{*}$ monomers in "living" radical polyermerizations. Macromolecules, 30(17), 5192-5194.

[11] Carlmark, A., \& Malmström, E. (2002). Atom transfer radical polymerization from cellulose fibers at ambient temperature. Journal of the American Chemical Society, 124(6), 900-901.

[12] Wegmann, S., Medalsy, I. D., Mandelkow, E., \& Müller, D. J. (2013). The fuzzy coat of pathological human tau fibrils is a two-layered polyelectrolyte brush. Proceedings of the National Academy of Sciences of the United States of America, Vol. 110, No. 4, pp. E313-E323.

[13] Luca, G. D., Lazzerini, W., Dabirian, R., Orgiu, E., Liscio, A., Palermo, V., et al. (2011). Non-conventional processing and post-precessing methods for the nanostructuring of conjugated materials for organic electronics. Advanced Functional Materials, 21(7), 1279-1295.

[14] Magonov, S. N., \& Whangbo, M. (1994). Interpreting STM and AFM images. Advanced Materials, 6(5), 355-371.

[15] Kim, Y. J., Son, K., Choi, I. C., Choi, I. S., Park, W. L., \& Jang, J. I. (2011). Exploring nanomechanical behavior of silicon nanowires: AFM bending versus nanoindentation. Advanced Functional Materials, 21(3), 279-286.

[16] Bayerl, D. J., \& Wang, X. (2012). Three-dimensional Kelvin probe microscopy for characterizing in plane piezoelectric potential of laterally deflected $\mathrm{ZnO}$ micro-/nanowires. Advanced Functional 


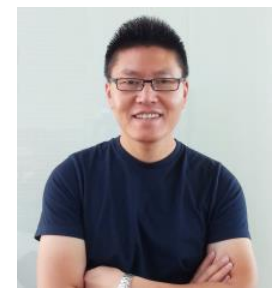

Xiaoqiang Yang got his joint Ph.D degree in polymer chemistry and physics at Sun Yat-Sen University in China and Tokyo Institute of Technology in Japan in July 2008. From Sept. 2008 to Sept. 2010, he worked at University of Wisconsin-Milwaukee in US as a postdoctoral fellow to conduct the interdisciplinary research about drug/gene/contrast agent delivery system. From 2011 to present, he joined prof. Donald E. Brooks' lab at University of British Columbia in Canada as a well-rounded research scientist, where he has privilege to investigate the interactions between biomaterials and blood.

His research interests and activities focus on the design, synthesis and application of functional biopolymers. In particular, he is interested in the interactions of these materials and their supramolecular assembles with biological systems to serve as new functional biomaterials and potential therapeutics. He has published more than thirteen peer-reviewed papers as the first author including ACS Nano, Biomaterials, Polymer, Polymer Chemistry, etc.

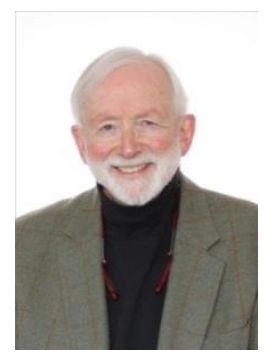

Donald E. Brooks is a physical biochemist with a background in biophysics, polymer science and surface chemistry and currently professor in the Departments of Pathology \& Laboratory Medicine and Chemistry at the University of British Columbia. He served as an UBC's associate vice president from 2001 to 2011 and was a founding member of the Centre for Blood Research in which they work. His current research focuses on in surface and polymer chemistry, particularly in developing polymer constructs for biomedical applications including blood compatible materials, blood plasma protein substitutes and drug delivery based on hyperbranched polyglycerol, a relatively new material to which they have made substantive contributions. Their work on choline phosphate based cell surface binding opens a new and exciting area of research, in the current case focusing on the development of bioadhesive materials for blood sealant applications.

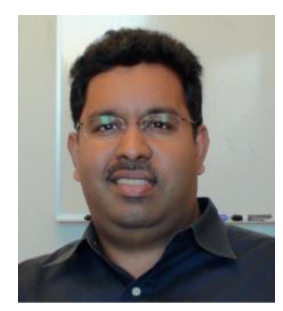

Jayachandran Kizhakkedathu is an associate professor in the Department of Pathology and Laboratory Medicine and in the Department of Chemistry at the University of British Columbia. He is a health research scholar for Michael Smith Foundation at the Centre for Blood Research (CBR). His research program at CBR (www.cbr.ubc.ca) is based on tailoring the molecular level interactions of synthetic polymers with biological systems to design novel biomaterials in a translational setting. They take an integrative, interdisciplinary approach with an understanding of the pathophysiology of diseases and advanced polymer synthesis in combination with well-designed biological assays and animal models towards the discovery of novel polymers as therapeutics, and technologies for biomedical and clinical use.

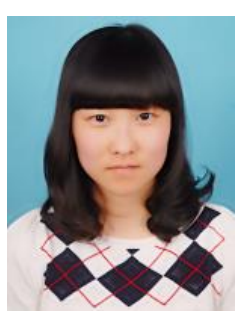

Na Li got the master degree in polymer chemistry and physics at Shaanxi University of Science and Technology in China. She started her Ph.D training from Sept. 2011 in materials physics and chemistry at Zhengzhou University in China, and began to study at Centre for Blood Research, University of British Columbia in Canada, as a joint Ph.D student in Nov. 2013. Her research project now is about biocompatible and blood compatible materials, such as CP and CP-based polymer brushes. 